Article

\title{
Phase Diagrams of Fatty Acids as Biosourced Phase Change Materials for Thermal Energy Storage
}

\author{
Clément Mailhé ${ }^{1, *}$, Marie Duquesne ${ }^{2}\left(0\right.$, Elena Palomo del Barrio ${ }^{3}$, Mejdi Azaiez ${ }^{2}$ and \\ Fouzia Achchaq 1 \\ 1 Université de Bordeaux, CNRS, I2M Bordeaux, Esplanade des Arts et Métiers, F-33405 Talence CEDEX, \\ France; fouzia.achchaq@u-bordeaux.fr \\ 2 Bordeaux INP, CNRS, I2M Bordeaux, Esplanade des Arts et Métiers, F-33405 Talence CEDEX, France; \\ marie.duquesne@enscbp.fr (M.D.); mejdi.azaiez@enscbp.fr (M.A.) \\ 3 CIC EnergiGUNE, Parque Tecnológico de Álava, Albert Einstein, 48. Edificio CIC, 01510 Miñano, Álava, \\ Spain; epalomo@cicenergigune.com \\ * Correspondence: clement.mailhe@u-bordeaux.fr
}

Received: 15 February 2019; Accepted: 6 March 2019; Published: 14 March 2019

check for updates

Featured Application: A potential application of this work consists of accelerating the screening step required for the study and selection of promising binary systems of phase change materials for thermal energy storage at medium temperature.

\begin{abstract}
Thermal energy storage is known as a key element to optimize the use of renewable energies and to improve building performances. Phase change materials (PCMs) derived from wastes or by-products of plant or animal oil origins are low-cost biosourced PCMs and are composed of more than $75 \%$ of fatty acids. They present paraffin-like storage properties and melting temperatures ranging from $-23^{\circ} \mathrm{C}$ to $78^{\circ} \mathrm{C}$. Therefore, they could be appropriate for latent heat storage technologies for building applications. Although already studied, a more detailed exploration of this class of PCMs is still required. In this frame, a screening of fatty acids and of their related binary systems must be performed. The infrared thermography method (IRT), already used for the fast estimation of simple phase diagrams $(\sim 2 \mathrm{~h})$, appears to be best suited to achieve this goal. IRT method applicability to the more complex fatty acids phase diagrams is hence studied in this work. A phase diagram comprising more than a hundred data sets was obtained for the palmitic acid-stearic acid binary system. The reliability of the results is assessed by comparison to differential scanning calorimetry (DSC) measurements or results from other standard methods presented in literature and to a solid-liquid equilibrium thermodynamic model.
\end{abstract}

Keywords: thermal energy storage; phase change materials; fatty acids; phase diagrams estimation; infrared thermography (IRT); polymorphism

\section{Introduction}

In France, the building sector is responsible for about $25 \%$ of $\mathrm{CO}_{2}$ emissions and $46 \%$ of energy consumption [1]. Thermal energy storage and distribution systems have been identified as efficient means of mobilizing renewable and recuperation renewable energies to improve building performances (particularly for heating and domestic hot water supply) [2-4]. Their uses are expected to be multiplied by 5 by 2030. Their developments are therefore a major challenge in the years to come.

Phase change materials (PCMs) used for the thermal energy storage represent an important class of materials which can substantially contribute to an efficient use and conservation of waste heat and solar energy in the building sector. Different groups of materials have been investigated during the 
technical evolution of phase change materials, including inorganic systems (salt and salt hydrates and even clathrate hydrates) and organic compounds such as paraffin waxes, esters, and polymeric materials or fatty acids (FA) [4-9]. These latter are promising PCM candidates. Indeed, not only do they represent more than 75\% of PCMs derived from wastes or by-products of plant or animal oils, but they also have advantageous thermal properties and energy densities for a limited cost [10]. Therefore, the applicability of FA as low-cost biosourced PCMs is studied in the perspective of limiting $\mathrm{CO}_{2}$ emissions and favoring renewable energy sources.

A screening step is required in order to select the most suitable fatty acids as well as their related binary systems. The most common methods used to establish the materials phase diagrams are differential scanning calorimetry (DSC) and differential thermal analysis (DTA), but the determination of a reliable phase diagram via these standard methods is very time-consuming and poorly adapted to screening procedures.

Our objective is to use the innovative method based on infrared thermography (IRT method) in the frame of a faster preliminary estimation of the phase diagrams, in a few hours only. This method was developed in the framework of the European FP7 Research Project SAM.SSA (2012-2015) and has been validated for the assessment and the study of simple phase diagrams of sugar alcohols (eutectic transitions) [11]. Our goal is now to adapt this method to fatty acids systems presenting more complex phase diagrams. To do so, we chose the palmitic acid (PA) and stearic acid (SA) binary system as an example because its transition temperature fit the aimed applications and its phase diagram includes eutectic, peritectic, and metatectic transitions. The results received with the IRT method for this binary system have been compared with the experimental data obtained using standard methods and with those collected from literature. The results have also been compared with a numerical thermodynamic model especially adapted in this work to PA, SA, and their polymorphisms.

\section{Materials and Methods}

The IRT method for the determination of phase diagrams is a dynamic method correlating phase transitions with the emissivity changes of a studied system. When the material of interest starts to crystallize/melt, an abrupt change in the emissivity is expected, leading to a change in the trend of the Digital-Level (DL) signals (see more details in Reference [11]). Once the phase change process is finished, the emissivity evolves steadily and the evolution of the signal with temperature is uniform again.

\subsection{Samples}

The binary system studied in this work consists of palmitic acid (PA) and stearic acid (SA). These materials belong to the vegetable oils considered as waste disposal that could even replace petroleum-based polymers, as explained in Reference [12]. Information regarding those materials and their properties is given in Table 1.

Table 1. General information about the fatty acids used in this study and provided by Sigma-Aldrich (St. Louis, MO, USA).

\begin{tabular}{ccc}
\hline & Palmitic Acid & Stearic Acid \\
\hline CAS number & $57-10-3$ & $57-11-4$ \\
Formula & $\mathrm{C}_{16} \mathrm{H}_{32} \mathrm{O}_{2}$ & $\mathrm{C}_{18} \mathrm{H}_{36} \mathrm{O}_{2}$ \\
Molar mass $(\mathrm{g} / \mathrm{mol})$ & 256.43 & 284.48 \\
$T_{m}\left({ }^{\circ} \mathrm{C}\right)$ in this work & 61.5 & 68.9 \\
$T_{m}\left({ }^{\circ} \mathrm{C}\right)$ from $[13-15]$ & $62.4,62.4,62.3$ & $70,69,70.8$ \\
$\Delta_{m} H(\mathrm{~J} / \mathrm{g})$ & 204 & 222,214 \\
$\Delta_{m} h(\mathrm{~J} / \mathrm{mol})$ & 52312 & 63155,60879 \\
Purity & $99 \%$ & $98,5 \%$ \\
Price $(\mathrm{USD} / \mathrm{kg})[16]$ & $0.7-0.83$ & $0.72-0.87$ \\
\hline
\end{tabular}


Droplets of the PA-SA binary system, each with a specific molar fraction of both components, are deposited on an aluminum plate and regularly spaced so that the heat released/absorbed by a droplet does not interfere with the others. The droplet composition ranges from 0 to a $100 \%$ of PA with a step increment of $1 \%$ in order to have a significant amount of data. Those samples were prepared by weighing each component $(\sim 3 \mathrm{mg})$ using a Mettler Toledo scale with a weighing accuracy of $\pm 0.03 \mathrm{mg}$.

\subsection{Experimental Setup}

The experimental setup used for the IRT phase diagram estimation is similar to the one described in Reference [11] (see Figure 1). An aluminum plate is placed under the infrared camera (IR camera FLIR X6580 sc, Detection window: $1.5-5 \mu \mathrm{m})$. This plate is coupled with a thermal resistance, a cooling element, and a thermocouple allowing to record, measure, and control the temperature of the samples. This thermocouple is glued to the aluminum plate using silver paint and insulated from its environment with insulating foam.

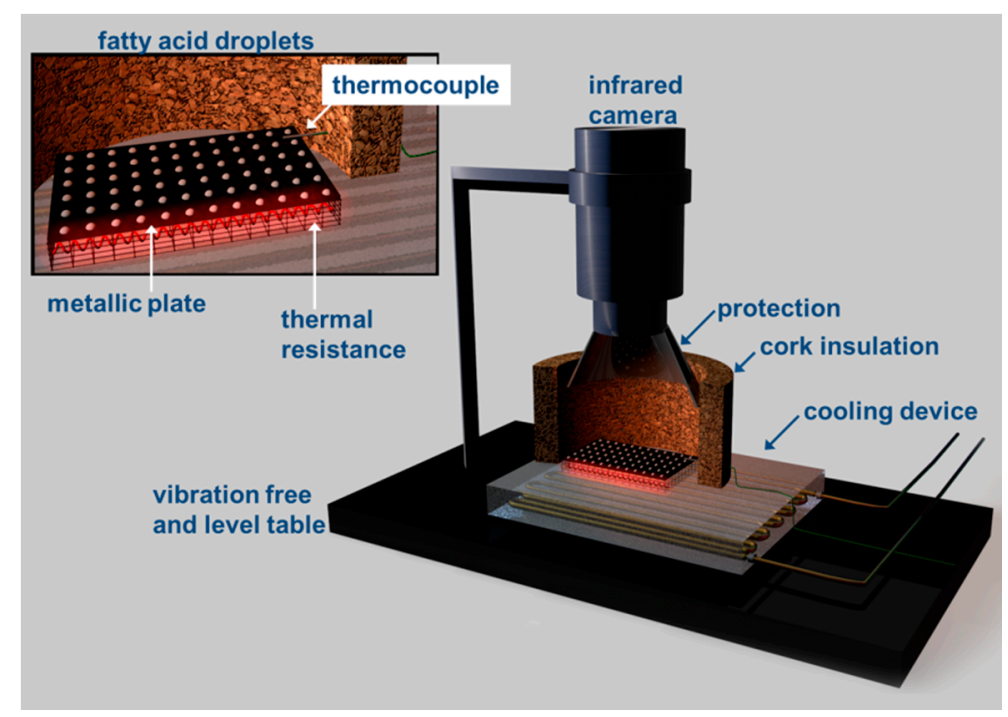

Figure 1. Sketch of the experimental setup.

\subsection{Experiment Protocols}

A heating/cooling ramp of $1{ }^{\circ} \mathrm{C} / \mathrm{min}$ is applied on the same principle as the DSC analysis performed for validation. The plate being highly conductive, the heating rate being low, and the droplets being small ( $\sim 5 \mathrm{~mm}$ diameter), the temperature of the plate is assumed to be uniform and the temperature of the droplets is assumed to always be equal to the plate one. Therefore, the thermocouple placed on the plate is assumed to record the temperature of each droplet at any time and allows the association of a signal variation to a specific transition temperature.

During the heating cycle, the IR camera simultaneously records the photonic flux emitted by each droplet placed on the aluminum plate. Its acquisition rate is set to $7 \mathrm{~Hz}$ to match the best compromise between the amount of gathered data and the processing time.

\subsection{Image Treatment and Data Processing}

The first step of the data processing consists of identifying the pixels corresponding to each individual droplet in the recorded raw infrared images. The associated DL signals are then extracted, which allows to reduce the whole set of raw images to one single image by averaging the DL signals on time. This average image captures main information regarding the position of the droplets on the plate. Their segmentation by thresholding produces a new binary image in which all pixels unambiguously associated to the droplets are identified by a single value (1) and the rest of the pixels (background) are all 0-valued. The connected components of the binary image foreground are then split in a set of 
disjoined elementary objects (individual droplets). This is done using the general procedure outlined in Reference [11]. Once elementary objects have been conveniently labelled (each droplet on the plate is identified by a different arbitrary color), the DL signals associated to each droplet can then be easily retrieved. In the second step, a droplet-by-droplet analysis is carried out to identify the temperature at which each droplet undergoes a transition. As a droplet can have hundreds of pixels (depending on the size of the droplet), the disparity in the signal of each plotted pixel makes the determination rather difficult, as can be seen in Figure 2.

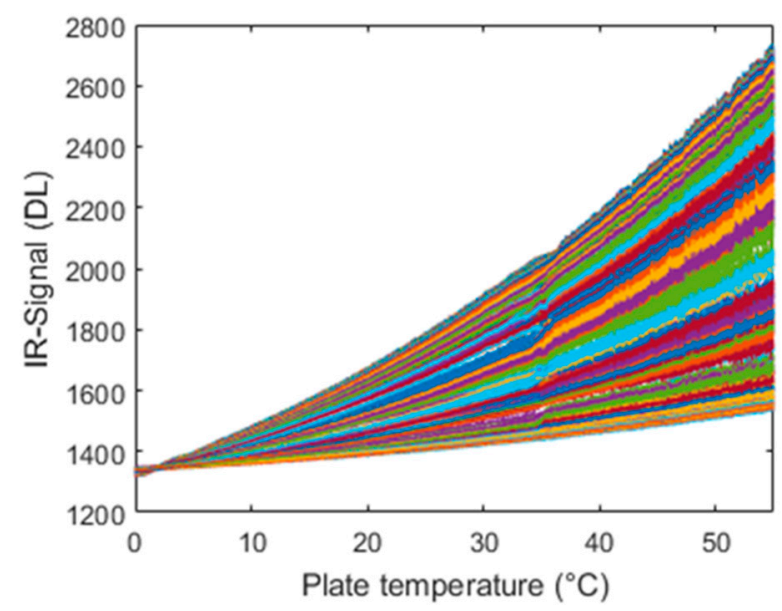

Figure 2. Example of recorded Digital-Level (DL) signal for each pixel of a droplet.

To overcome this difficulty, a singular value decomposition (SVD) technique described in Reference [11] was applied to our data. The determination of the transition lines is now reduced to the identification of abrupt changes in three of the first four time-eigenfunctions $Z_{i}(t)$ for each droplet, as can be seen in Figure 3.

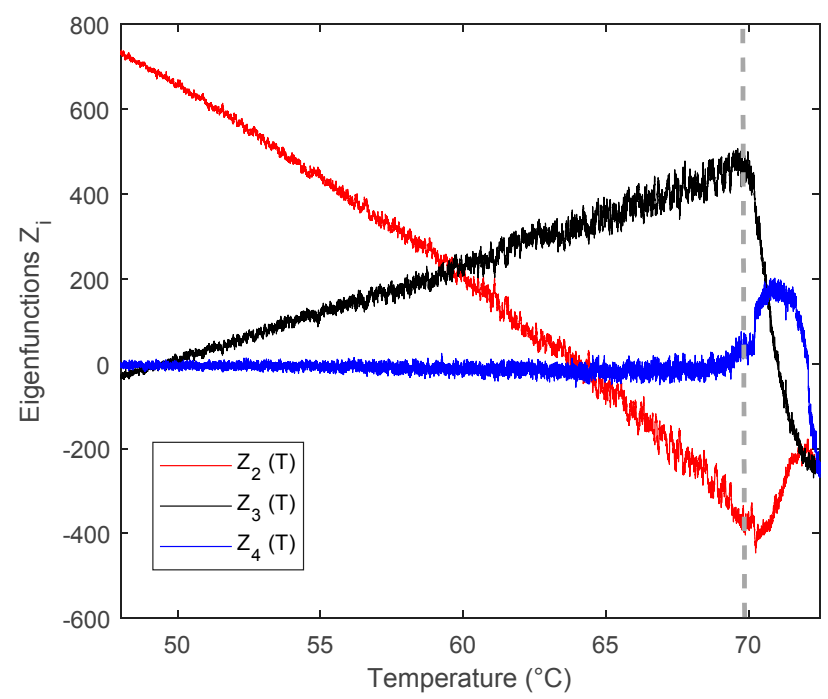

Figure 3. Temperature behavior of three of the first four eigenfunctions for a droplet of stearic acid (SA).

\subsection{Experimental and Theoretical Validations}

The results obtained using the IRT method were first confronted with the literature data, then with the experimental ones collected from a differential scanning calorimetry (DSC) analysis. The DSC experiments were performed with samples of the same composition as the droplets used for the IRT experiments. 
Moreover, the results were also compared with a thermodynamic model especially developed to estimate the liquidus line of the organic PCMs' binary systems presenting a peritectic transition.

\subsubsection{DSC Analysis}

DSC analysis was performed at ambient atmosphere using a DSC 131 supplied by Setaram (Caluire, France). Two series of experiments were performed, one with a $1{ }^{\circ} \mathrm{C} / \mathrm{min}$ heating ramp for a preliminary estimation and the other one at a rate of $0.3^{\circ} \mathrm{C} / \mathrm{min}$ for more accurate transition detections. As for the studied compositions, 8 molar fractions of PA-SA $(0 / 100,15 / 85,30 / 70,40 / 60,55 / 45,70 / 30$, $86 / 14$, and 100/0 in molar percentages of PA/SA) were chosen to assess the best phase diagram possible in the least amount of time (knowing that this procedure can be really time-consuming when accurate results are required). The molar fraction was chosen to depict a coherent phase diagram in a minimal amount of data while providing insightful information regarding detected transitions using the IRT method and providing additional/complementary data in comparison with literature [15]. The phase transitions are generally related to the heat flux evolution as a function of temperature. A peak appears when a specific process, such as melting or crystallization, occurs. Transition temperatures on thermograms are identified according to the guidelines recommended in Reference [17]. The onset temperature is a better indicator of the transition process beginning when a transition peak starts and ends with a flat baseline. In such cases, the transition temperature hence corresponds to the onset temperature on the thermogram. However, multicomponent mixtures are often characterized by the presence of several transitions occurring either successively or almost simultaneously. A reliable onset temperature cannot always be extracted in those multipeak thermograms. Transition temperatures are therefore considered as the peak temperatures in those situations. Additionally, if a clear reading is still impossible, it is recommended, if available, to use complimentary techniques (differential thermal analysis, $\mathrm{X}$-ray powder diffraction, etc.) to enhance the results interpretation.

\subsubsection{Thermodynamic Modeling of the Solid-Liquid Equilibrium}

Most models aiming at modeling the solid-liquid equilibrium of organic compounds' binary systems are either limited to the depiction of simple eutectic systems or rely on experimental data to fit unknown parameters. A model was implemented using Matlab Software (MathWorks, Natick, MA, USA) for this study. It is a combination of the models described in Reference [18] accounting for the polymorphic behavior of fatty acids and in Reference [19] introducing means of modeling the effect of the peritectic transition on the liquidus line.

When a pure component is cooled/heated, different solid-solid transitions may occur. Most models tend to neglect those transitions because of their weak enthalpy value. However, fatty acids show a strongly polymorphic behavior. This polymorphic behavior is highly influenced by the cooling/heating rate and the nature of the liquid mixture made of both components. The polymorphism of even saturated fatty acids was studied in Reference [20]. The authors showed that two main parameters can influence the materials behavior and the resulting properties: The polarity in solution of the multicomponent mixture and the crystal growth kinetics. It is therefore primordial to consider, for the material properties, the ones that match the experimental conditions. As the DSC analyses in Reference [15] and our experiment with the IRT method were performed at a $1{ }^{\circ} \mathrm{C} / \mathrm{min}$ rate, a slow crystal growth rate is assumed. Additionally, as PA and SA both consist in long carbon chains with only one polar group at the end, the blend of both is considered to be weakly polar [21]. The polymorphic transitions temperatures and enthalpies considered for this study are given in Table 2 . They were extracted from Reference [20] considering the assumptions previously stated. 
Table 2. Values of temperatures of transitions, molar enthalpies of transitions and mass molar enthalpies used in the model to take into account the polymorphic behavior of palmitic and stearic acids [20].

\begin{tabular}{ccc}
\hline & Palmitic Acid & Stearic Acid \\
\hline \multirow{2}{*}{$T_{t r}\left({ }^{\circ} \mathrm{C}\right)$} & \multirow{2}{*}{51.6} & 51.3 \\
& & 52.8 \\
& & 58.5 \\
\hline \multirow{3}{*}{$\Delta_{t r} h(\mathrm{~J} / \mathrm{mol})$} & \multirow{2}{*}{2600} & 5400 \\
& & 5700 \\
& & 2800 \\
\hline \multirow{2}{*}{$\Delta_{t r} H(\mathrm{~J} / \mathrm{g})$} & \multirow{2}{*}{10.14} & 18.98 \\
& & 20.04 \\
& & 9.84 \\
\hline
\end{tabular}

As most models rely on the assumption that both pure components are not miscible in the solid phase, the peritectic transition represents a major challenge in the modeling of solid-liquid equilibrium of complex organic mixtures. Therefore, the results may differ significantly depending on this assumption.

To overcome this issue, the peritectic compound can be seen as a completely new compound obtained through a reaction of both pure components for a specific stoichiometry. This stoichiometry cannot be known prior to the establishment of the phase diagram. However, given the phase diagram plotted by the authors in Reference [15], a 0.4-0.6 molar ratio can be assumed for this binary system. Only one additional parameter is required to model the liquidus line with this assumption. This parameter is the equilibrium constant of the involved chemical reaction. It assumes that an actual reaction occurs, which is not the case for this system. The peritectic compound is merely a crystal composed of both pure components in a fixed stoichiometric. Therefore, the value computed for this parameter can be considered as an adjustable parameter rather than a meaningful physical value for this reaction. Its purpose is here to allow the best fit between the model and experimental data.

The following equation details the model used in this work to compute the solid-liquid equilibrium of the peritectic compound:

$$
\sum_{i} \ln \left(x_{i} \gamma_{i}^{L}\right)=\sum_{i} v_{i}\left(\frac{\Delta_{m} h_{i}}{R}\left(\frac{1}{T_{m}^{i}}-\frac{1}{T}\right)+\sum_{t r=1}^{n}\left(\frac{\Delta_{t r} h_{i}}{R}\left(\frac{1}{T_{t r}^{i}}-\frac{1}{T}\right)\right)\right)-\frac{\Delta_{r} G^{0}}{R T},
$$

where $i$ identifies the pure component, $L$ characterizes the liquid phase, $x_{i}$ is the liquid phase molar fraction of $i, \gamma_{i}$ is the liquid phase activity coefficient of $i, R$ is the universal gas constant, $T_{m}$ and $\Delta_{m} h$ are the melting temperature and enthalpy, respectively, $T_{t r}$ and $\Delta_{t r} h$ are the polymorphic transition temperatures and enthalpies for the component $i, v_{i}$ is the stoichiometric coefficient associated to the reaction forming the peritectic compound, and $\Delta_{r} G^{0}$ is Gibbs-free energy of the peritectic reaction.

The model used for the depiction of the solid-liquid equilibrium of the PA-SA binary system part, not influenced by the peritectic reaction, is detailed in Equation (2):

$$
\ln \left(x_{i} \gamma_{i}^{L}\right)=\frac{\Delta_{m} h_{i}}{R}\left(\frac{1}{T_{m}^{i}}-\frac{1}{T}\right)+\frac{\Delta_{t r} h_{i}}{R}\left(\frac{1}{T_{t r}^{i}}-\frac{1}{T}\right)
$$

It is a common form as described in References [22,23]. The calculation of the liquid activity coefficient $\gamma_{i}$ is performed using the UNIFAC-Dortmund method [24], and for each value of $x_{i}$, the transition temperature satisfying the equation is calculated. It implies knowing the melting temperature and the enthalpy values as well as the polymorphic transition temperatures and enthalpies for each pure component. For our model, the considered melting properties values are presented in Table 1 and the polymorphic properties in Table 2 . 


\section{Results and Discussion}

The results of the IRT experiment carried out on the binary system of PA and SA are reported in Figures 4 and 5 and in Table 3.

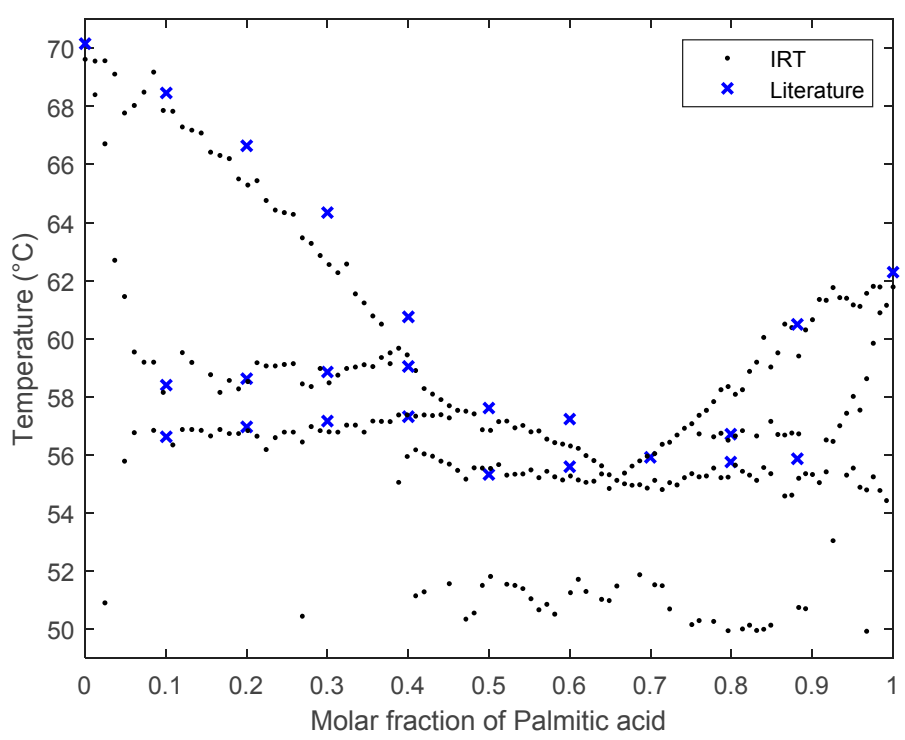

Figure 4. Phase diagrams comparison between the infrared thermometry method (IRT) experiment and literature data [15] for the palmitic acid (PA)-SA binary system.

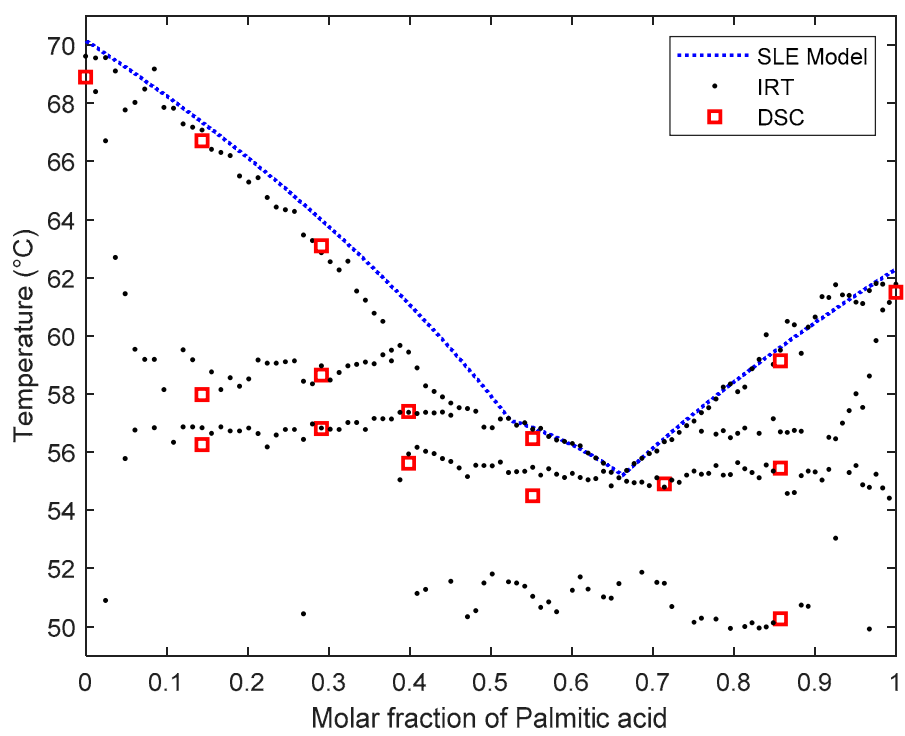

Figure 5. Comparison between the IRT experiment, differential scanning calorimetry (DSC) analysis and the numerical approach for the (PA-SA) binary system. 
Table 3. Comparison between experimental and literature properties of the PA-SA binary mixture.

\begin{tabular}{ccc}
\hline & This Work (IRT) & Literature \\
\hline Eutectic point (molar \%PA) & & $66.6 \%[13]$ \\
& $67 \%$ & $66.6 \%[14]$ \\
& & $70 \%[15]$ \\
\hline & & $52.3[13]$ \\
$T_{\text {eutectic }}\left({ }^{\circ} \mathrm{C}\right)$ & 55.12 & $52[14]$ \\
& & $55.75[15]$ \\
\hline$T_{\text {peritectic }}\left({ }^{\circ} \mathrm{C}\right)$ & 57.37 & $57.05[15]$ \\
\hline
\end{tabular}

The phase diagram plotted from the IRT experiments is compared to the experimental data collected from Reference [15] in Figure 4. The obtained diagram is consistent with the literature one [15]. The method succeeds in detecting the liquidus line, the eutectic, the peritectic, and even the metatectic transitions. On the right part of the phase diagram of the phase diagram, a transition is detected around $56.7^{\circ} \mathrm{C}$. This latter would most likely be a metatectic one given the overall shape of the phase diagram and considering results found in literature for other fatty acids' binary systems $[15,25,26]$. The presence of a metatectic transition implies the existence of a solid solubility domain. This domain is rather difficult to detect using the standard methods. In Reference [15], its location was estimated using Tamman plots but not backed by measurements performed using DSC or other methods. Moreover, the phase diagram presented in Figure 4 also shows other transitions on the left-hand side. Those transitions are coherent with the position of this potential solid solubility domain which would be a great advantage for the simple and fast IRT method in comparison with standard methods.

The sample composition corresponding to the eutectic point is within the range of that found in the literature (see Table 3). Additionally, the droplet deposition on the aluminum plate and the numerical data treatment are not automated. It induces slight uncertainties during the sample preparation (size and composition) as well as during the data interpretation. Those user-induced uncertainties could explain some noticeable irregularities between adjacent points in the phase diagram (loss of continuity, missing data, eigenfunctions misinterpretation, etc.). Moreover, it appears that the temperature values related to the liquidus line are slightly underestimated with the IRT method. However, our DSC results are consistent with the IRT ones, as shown in Figure 5. Hence, this deviation could more likely be due to differences in pure components purities, inaccuracies in the samples' composition, and/or the user's interpretation of the thermograms.

Despite these slight observed discrepancies between data, Figure 5 validates what is observed in Figure 4. The DSC thermograms for the sample with $86 \%$ PA-14\% SA composition show that a phase transition occurs in the $57-58{ }^{\circ} \mathrm{C}$ range. However, a clear reading on the transition temperature could not be extracted and was therefore left aside to avoid including erroneous data. The IRT phase diagram coincides with the one obtained with the standard methods, suggesting that the IRT method is reliable to estimate the complex phase diagrams of fatty acids' binary systems. It provides the compositions and the temperatures at which several transitions occur (with the previously mentioned uncertainties). Moreover, this method allows the determination of approximately one hundred data sets in a single heating/cooling cycle (one hour to estimate this phase diagram), whereas the standard methods such as DSC or DTA require at least two to three cycles for a single composition (a few weeks to estimate this phase diagram). The results obtained using the IRT method are consistent with the DSC ones. Additionally, a solid-solid transition occurring around $50.5^{\circ} \mathrm{C}$ was measured via DSC. This transition is consistent with IRT results, but it has not yet been found in enough samples to confirm the occurrence of such transition with certainty. It is also interesting to note that this temperature is close to considered polymorphic transitions of both pure compounds. A major step to be mentioned is also the potential use of the IRT method of the detection of transitions that could be associated to the limits of the solid solubility domain. A metatectic transition, not fully validated with DSC measurements (as mentioned previously), is also found in the right part of the phase diagram. Considering the phase 
diagrams of other fatty acids' binary systems, such a transition could however be expected. Further works are therefore needed to confirm the results obtained with the IRT method (validation of the solid solubility domain detection and of the polymorphic transitions detection). Additionally, and as mentioned previously, efforts are expected to automatize the transitions detection and the sample preparation in order to increase the IRT method accuracy.

In addition, the adjusted thermodynamic model is able to highlight the effect of the peritectic transition on the liquidus line, providing a good fit with IRT data. However, the equilibrium constant value giving the best approach is still tricky to find. Moreover, depending on the properties chosen for the pure components and on the applied cooling/heating rates, completely different diagrams may be obtained for the same binary system. Another limitation to the modeling of solid-liquid equilibrium for such systems is the occurrence of a metatectic transition and of solid solutions. Indeed, as the model assumes immiscibility of the components in the solid phase, it is in contradiction with the presence of a solid solubility domain. Although its shape and its location are coherent with the experimental data, a noticeable deviation occurs in the 0.3-0.5 molar fraction range. This deviation seems to be growing and coincides with the increase of the solid miscibility of both compounds. Essentially, the problem could be treated in the same way as described in Section 2.5.2 but by considering, this time, a solid solution or metatectic compound formed from a reaction with a variable stoichiometry. If this solid solubility domain can in fact be determined using the IRT method, then the stoichiometry of the metatectic compound can be known and the solid-liquid equilibrium can hence be computed optimally by adjusting the value of the Gibbs-free energy of reaction.

\section{Conclusions}

The IRT method allows the estimation of a preliminary phase diagram of the PA-SA binary system. It was able to detect all transitions either found in literature or with DSC. Additional transitions were also detected, but their occurrence could not be proven with DSC analysis. Efforts are expected to confirm the detection of the solid solubility domain and the polymorphic transitions, as their detection could represent a major feat. Although its accuracy is still to be improved, the IRT method has proven to be adapted even in the case of more complex phase diagrams, such as the ones of binary systems of fatty acids. With the amount of data gathered through this experiment in such a limited time $(\sim 1 \mathrm{~h})$, the efficiency of the IRT method for the screening of new materials is undoubtful. Further works are expected in order to constantly improve this innovative method. A robot is under development for the droplet deposition automation in order to obtain reproducible droplets shape and size, and efforts are also made to automatize data treatment, which will eventually provide optimized estimations as systems are being studied. With its accuracy and efficiency increasing, it is expected to detect an increasingly more complex transition and could eventually provide complementary information regarding the thermal properties of studied systems (transition enthalpy, specific heat).

Author Contributions: Conceptualization, E.P.d.B. and M.D.; methodology, E.P.d.B., M.D., and C.M.; software, C.M., M.D., and E.P.d.B.; validation, C.M., M.D., and F.A.; formal analysis, C.M., M.D., E.P.d.B., F.A., and M.A.; investigation, C.M.; resources, C.M., M.D., E.P.d.B., F.A., and M.A.; data curation, E.P.d.B., C.M., and M.D.; writing—original draft preparation, C.M.; writing—review and editing, C.M., M.D., and F.A.; visualization, C.M., M.D., and F.A.; supervision, M.A. and M.D.; project administration, M.A., E.P.d.B., and M.D.; funding acquisition, E.P.d.B. and M.D.

Funding: This work is carried out in the frame of SUDOKET project and is co-funded by the Interreg Sudoe Programme through the European Regional Development Fund (ERDF).

Acknowledgments: We would like to thank CNRS for promoting the I2M Bordeaux-CIC exchanges in the framework of the PICS PHASE-IR project. We also want to thank the "Illustration 3D: 25 images communication" company.

Conflicts of Interest: The authors declare no conflict of interest. 


\section{References}

1. International Energy Agency (2019). Buildings: Tracking Clean Energy Progress. Available online: https: / / www.iea.org/tcep/buildings/ (accessed on 2 March 2019).

2. Agyenim, F.; Hewitt, N.; Eames, P.; Smyth, M. A review of materials, heat transfer and phase change problems formulation for latent heat thermal energy storage systems. Renew. Sustain. Energy Rev. 2010, 14, 615-628. [CrossRef]

3. Duquesne, M.; Toutain, J.; Sempey, A.; Ginestet, S.; Palomo del Barrio, E. Modeling of a non-linear thermochemical energy storage by adsorption on zeolites. Appl. Therm. Eng. 2014, 71, 469-480. [CrossRef]

4. Alva, G.; Lin, Y.; Fang, G. An overview of thermal energy storage systems: A review. Energy 2018, 144, 341-378. [CrossRef]

5. Zalba, B.; Marin, J.M.; Cabeza, L.F.; Mehling, H. Review on thermal energy storage with phase change: Materials, heat transfer analysis and applications. Appl. Therm. Eng. 2003, 23, 251-283. [CrossRef]

6. Yuan, Y.; Zhang, N.; Tao, W.; Cao, X.; He, Y. Fatty acids as phase change materials: A review. Renew. Sustain. Energy Rev. 2014, 29, 482-498. [CrossRef]

7. D'Avignon, K.; Kummert, M. Experimental assessment as a phase change material storage tank. Appl. Therm. Eng. 2016, 99, 880-891. [CrossRef]

8. Castellani, B.; Morini, E.; Filipponi, M.; Nicolini, A.; Palombo, M.; Cotana, F.; Rossi, F. Clathrate hydrates for thermal energy storage in buildings: Overview of proper hydrate-forming compounds. Sustainability 2014, 6, 6815. [CrossRef]

9. Ning-Wei Chiu, J.; Martin, V.; Setterwall, F. A Review of Thermal Energy Storage Systems with Salt Hydrate Phase Change Materials for Comfort Cooling. In Proceedings of the 11th International Conference on Thermal Energy Storage, Stockholm, Sweden, 14-17 June 2009.

10. Maximo, G.J.; Costa, M.C.; Coutinho, J.A.P.; Meirelles, A.J.A. Trends and Demands in the Solid-liquid Equilibrium of Lipidic Mixtures. RSC Adv. 2014, 4, 31840-31850. [CrossRef]

11. Palomo Del Barrio, E.; Cadoret, R.; Daranlot, J.; Achchaq, F. Infrared thermography method for fast estimation of phase diagrams. Thermochim. Acta 2016, 625, 9-19. [CrossRef]

12. Lligadas, G.; Ronda, J.C.; Galia, M.; Cadiz, V. Renewable polymeric materials from vegetable oils: A perspective. Materials Today 2013, 16, 337-343. [CrossRef]

13. Pielichowska, K.; Pielichowski, K. Phase change materials for thermal energy storage. Prog. Mater. Sci. 2014, 65, 67-123. [CrossRef]

14. Kenisarin, M.M. Thermophysical properties of some organic phase change materials for latent heat storage. A review. Sol. Energy 2014, 107, 553-575. [CrossRef]

15. Costa, M.C.; Sardo, M.; Rolemberg, M.P.; Meirelles, A.J.A.; Coutinho, J.A.P.; Ribeiro-Claro, P.; Krähenbühl, M.A. The Solid-liquid Phase Diagrams of Binary Mixtures of Even Saturated Fatty Acids. Chem. Phys. Lipids 2009, 160, 85-97. [CrossRef]

16. Oleoline (2018). OLEOCHEMICALS'MARKET PRICES. Available online: http:/ /www.hbint.com/datas / media/5b7d6eb55e3e88fa6677fa28/two-weekly-oleochimicals.pdf (accessed on 2 March 2019).

17. Boettinger, W.J.; Kattner, U.R.; Moon, K.W.; Perepezko, J. Chapter V-DTA and Heat-Flux DSC Measurements of Alloy Melting and Freezing. In Methods for Phase Diagram Determination; Elsevier Science Ltd.: Oxford, UK, 2007; pp. 151-221.

18. Maximo, G.J.; Costa, M.C.; Meirelles, A.J.A. The Crystal-T Algorithm: A New Approach to Calculate the SLE of Lipidic Mixtures Presenting Solid Solutions. Phys. Chem. Chem. Phys. 2014, 16, 16740-16754. [CrossRef]

19. Barbosa, D.F.; de Alcântara Pessôa Filho, P. On the Description of the Liquidus Line of Systems Presenting Peritectic Reactions. Fluid Phase Equilibria 2013, 337, 379-383. [CrossRef]

20. Moreno, E.; Cordobilla, R.; Calvet, T.; Cuevas-Diarte, M.A.; Gbabode, G.; Negrier, P.; Mondieig, D.; Oonk, H.A.J. Polymorphism of even saturated carboxylic acids from n-decanoic to n-eicosanoic acid. New J. Chem. 2007, 31, 947-957. [CrossRef]

21. Hoerr, C.W.; Balsto, A.W. The solubilities of the normal saturated fatty acids. II. J. Org. Chem. 1944, 9, 329-337. [CrossRef]

22. Maximo, G.J.; Carareto, N.; Costa, M.C.; dos Santos, A.O.; Cardoso, L.P.; Krähenbühl, M.A.; Meirelles, A.J.A. On the solid-liquid equilibrium of binary mixtures of fatty alcohols and fatty acids. Fluid Phase Equilibria 2014, 366, 88-98. [CrossRef] 
23. Maximo, G.J.; Aquino, R.T.; Meirelles, A.J.A.; Krähenbühl, M.A.; Costa, M.C. Enhancing the description of SSLE data for binary and ternary fatty mixtures. Fluid Phase Equilibria 2016, 426, 119-130. [CrossRef]

24. Gmehling, J.; Li, J.; Schiller, M. A modified UNIFAC model. 2. Present parameter matrix and results for different thermodynamic properties. Ind. Eng. Chem. Res. 1993, 32, 178-193. [CrossRef]

25. Costa, M.C.; Sardo, M.; Rolemberg, M.P.; Ribeiro-Claro, P.; Meirelles, A.J.A.; Coutinho, J.A.P.; Krähenbühl, M.A. The solid-liquid phase diagrams of binary mixtures of consecutive, even saturated fatty acids: Differing by four carbon atoms. Chem. Phys. Lipids 2009, 157, 40-50. [CrossRef] [PubMed]

26. Costa, M.C.; Rolemberg, M.P.; Meirelles, A.J.A.; Coutinho, J.A.P.; Krähenbühl, M.A. The solid-liquid phase diagrams of binary mixtures of even saturated fatty acids differing by six carbon atoms. Thermochim. Acta 2009, 496, 30-37. [CrossRef]

(C) 2019 by the authors. Licensee MDPI, Basel, Switzerland. This article is an open access article distributed under the terms and conditions of the Creative Commons Attribution (CC BY) license (http:/ / creativecommons.org/licenses/by/4.0/). 